\title{
Attitudes of parents towards measles and immunisation after a measles outbreak in an anthroposophical community
}

\author{
E Duffell
}

The introduction of mass immunisation against measles in the United Kingdom has seen the virtual disappearance of this disease. In some European countries with lower levels of vaccine coverage, however, deaths from measles are still common and the disease is associated with significant morbidity. ${ }^{1}$ In recent years adverse publicity surrounding the vaccine has depressed vaccine uptake in the United Kingdom. Parental attitude towards disease is known to be a powerful predictor of vaccine uptake. ${ }^{2}$ Indeed, reports from the Health Education Authority tracking studies of parental attitudes to vaccination suggest that confidence in the safety of MMR vaccine has fallen in parallel with vaccine uptake, ${ }^{3}$ and many parents now consider the vaccine to be a greater threat to their child's health than measles itself.

\section{Methods and Results}

An outbreak of measles among children from a predominantly un-immunised anthroposophical community in Gloucestershire provided an opportunity to explore parental views and study disease severity in this group. Anthroposophy is a spiritual movement that follows the teachings of Rudolf Steiner, an Austrian philosopher and scientist born in 1861. The term literally implies a wisdom about man, which is produced by the higher self in man and achieved through meditation and concentration. ${ }^{4}$ Anthroposophical medicine advocates freedom of choice in health care and natural remedies. This type of medical practice is in favour of letting the body experience certain infections and is against the overuse of antibiotics, antipyretics and certain immunisations. Many adherents oppose the measles vaccine because they believe children gain physical and mental robustness from natural measles infection, when supported by appropriate nursing care. Indeed, as Hanratty et al note "the avoidance of immunisation in these communities is more than a refusal to accept conventional medicine". 5

A postal questionnaire was sent to notified cases of measles from the $1997 / 8$ outbreak in Gloucestershire. A total of 126 questionnaires were returned, giving an overall response rate of $59 \%$. Among the respondents the mean age of the cases was 7.9 years, the mean time off school was 14 days, 76 consulted their general practitioner, 61 received prescribed medication and one child was admitted to hospital. Some $62 \%$ of these respondents reported a change in their child's personal development subsequent to the measles infection, with many claiming a strengthening and maturing of their child both mentally and physically. Of the cases, $87 \%$ were reported to have been previously unimmunised with the measles vaccine.

The main reasons cited for not being vaccinated included issues relating to the safety of the vaccine and its effectiveness. Many believed that measles is important for a child's development and had a "beneficial strengthening effect upon the child". Many respondents were also concerned about the long term side effects of the vaccine and the effects of the vaccine on the immune system. Friends and relatives were the main reported influences behind the parent's decision to immunise. Although $83 \%$ of respondents viewed measles as a serious infection, only three felt that their child's illness had changed their opinion on immunisation.

\section{Discussion}

The findings of low levels of morbidity associated with measles are similar to previous studies in the United Kingdom, ${ }^{2}$ and support the notion that measles is not a severe illness in most children. These cases were, however, in fit, well nourished children from a community that advocates a healthy lifestyle and there were insufficient numbers of cases to observe many of the rarer sequelae. Influencing parental attitudes by emphasising the severity of the illness was one tactic used to improve coverage in the run up to the 1994 measles-rubella vaccination campaign, which was successful in increasing vaccine coverage and preventing a predicted measles epidemic. The observations from this survey suggest a more appropriate tack for vaccination campaigners may be to emphasise the safety issues of the vaccine and its effectiveness in a balanced argument. The results also suggest that, rather than simply repeating the scare tactics used in previous campaigns, it may be worthwhile exploring the views of vaccine refusers in more detail and considering alternative approaches to promoting vaccination in such groups.

I am extremely grateful to Dr David Hunt for his continued support and assistance in the preparation of this paper. I would also like to thank Dr Wouter Havinga for his involvement in the survey and Dr Andy Ness for his constructive comments on an earlier draft of this paper.

Funding: none.

Conflicts of interest: none.

\footnotetext{
1 Begg N, Ramsay M, White J, et al. Media dents confidence in MMR vaccine. BMf 1998;316:561.

McGuire C, Bozoky Z, Sinkler P. Health update: immunisation. London: Health Education Authority, 1998.
} 
3 CDSC. MMR vaccine coverage falls in the United Kingdom Commun Dis Rep 1999;9:37.

Kingdom. Commun Dis Rep 1999;9:37. with an anthroposophical lifestyle. Lancet 1999;353:1485-8.
5 Hanratty B, Holt T, Duffell E, et al. UK measles outbreak in non-immune anthroposophic communities: the implications for the elimination of measles from Europe. Epidemiol Infect 2000;125:377-83.

\section{What's in the next issue}

\section{Future content}

See which articles have just been accepted for publication and preview the table of contents for the next issue a month before it is published

\section{www.jech.com}

